\title{
Impact of Covid-19 on Alterations in Food Habits and Lifestyle Behaviour of Indians: A Review
}

\section{Jyoti Singh ${ }^{1}$ and Nikita Wadhawan ${ }^{2 *}$}

${ }^{1}$ Research Scholar, Department of Food Science and Nutrition, MPUAT, Udaipur,

Rajasthan, India

${ }^{2}$ Assistant Professor, Department of Renewable Energy Engineering, MPUAT,

Udaipur, Rajasthan, India

*Corresponding Author: Nikita Wadhawan, Assistant Professor, Department of

Renewable Energy Engineering, MPUAT, Udaipur, Rajasthan, India.
Received: March 08, 2021

Published: April 27, 2021

(C) All rights are reserved by Jyoti Singh and

Nikita Wadhawan.

\begin{abstract}
People from all walks of life have been seriously influenced by the COVID-19 pandemic. The global dissemination of the disease to almost all areas of the world has provided the entire human population with immense health, economic, environmental and social challenges. One of the solutions proposed by the World Health Organisation (WHO) for minimising the spread of this deadly virus is lockdown. On March 22, 2020, India was swift to close its international boundaries and implement the world's biggest COVID lockdown. This Pandemic had affected every age group though, the time is more crucial: for children because of their increased exposure to television advertisements directed at the selling of junk foods, those in their adolescence because it is the age of habit formation and the incorrect habit developed during this period will begin to affect their adult life. In general, it could result in obvious epigenetic and/or phenotypic variation being passed on to subsequent generations, as well as significant dietary changes leading to increased obesity and hyperglycaemia in many countries. There is insufficient evidence on how this pandemic is impacting our food and lifestyle-related habits. Therefore, this study reviews impact of COVID-19 on alterations in food habits and lifestyle behaviour of Indians. These unprecedented circumstances provide an opportunity to better understand the negative impact of strict lockdown measures on lifestyles, and at the same time, help identify and initiate positive behavioural changes, which if consolidated, may improve chronic disease outcomes in the long run. It would be up to governments, communities, and academia to learn - and benefit from lessons learned from this pandemic - with the ultimate objective of better educating and promoting healthy lifestyles among communities.
\end{abstract}

Keywords: COVID-19; Pandemic; Lockdown; Dietary Changes; Immunity Boosters; Herbs; Lifestyle Behaviour; Stress; Sleep

\section{Introduction}

The outbreak COVID-19 was first reported in late December 2019 solely in the city of Wuhan, China. By the end of January 2020, World Health Organization (WHO) declared COVID-19 as a public health emergency of international concern and on March 11,2020 , WHO characterized this epidemiological phenomenon as a global pandemic. The first confirmed case of SARS-CoV-2 positive in India was reported on 30th January. The most common symptoms of Covid-19 are fever, cough and breathing issues.
In the severe cases, the symptoms may be worse with pneumonia leading to organ failure and death [1]. The data from various countries shows that this virus has infected people of all ages. However, older people and already sick (due to declining immune system) people with pre-existing medical conditions like diabetes, hypertension, cardiovascular disease and asthma appear to be vulnerable. The widespread dissemination of the disease to virtually all areas of the nation has been immense for the whole human so- 
ciety, health, educational, environmental and social challenges. The drastic growth in daily case counts, the government had drawn up plans to deal with a worsening of the pandemic in the country.

As a result, on March 24, 2020, India's government instituted a 21-day nationwide lockdown restricting the movement of the country's 1.3 billion people. Also, on 16 March, the union government declared a countrywide lock-down of schools and colleges. In addition, Ministry advisories such as AYUSH have suggested steps to help the public stay safe during pandemics. The Ministry has issued recommendations for practitioners on how to handle the pandemic, including immunity-boosting foods, warm water consumption, a balanced diet, and physical activity etc. to promote health. On April 2, 2020, the Government of India officially launched a COVID -19 monitoring framework called "Aarogya Setu" in an effort to protect the country's people [2]. The app aims to get people involved proactively in best relevant practices and follow advisories to cope well with an ongoing virus outbreak. Since the pace of life slowed due to the pandemic, India's large and diverse population faces unique challenges in terms of safe access to nutritious food, money, basic supplies, social care, and medicine to support their physical health.

Though these were vital to allay the spread of COVID-19, the effect of these restrictions on nutrition and lifestyle at home is innumerable [3]. These measures include insufficient access to shops, a poorer quality of food products due to discernible effects on family income, and overeating due to various reasons [4]. It has also resulted in a disruption of the daily schedule, which leads to boredom, which is related to increased energy usage. Stress from the global pandemic, as well as other factors such as hearing or reading about the COVID-19 in the media, causes sleep disturbances and a low mood, which contributes to overeating, especially of sugary comfort foods, which is referred to as "food craving" [5]. These simple carbohydrate-rich foods alleviate stress by increasing serotonin output, which has a positive impact on mood. This increases the total consumption of unhealthy food items over healthy food items, thus alarming the health of individuals. Moreover, prolonged confinement at home may induce an increased screen time and reduced outdoor time thus, augmenting sedentarism. All of these habits can lead to an increased risk of developing obesity and car- diovascular diseases, beyond a chronic state of inflammation that has been shown to increase the risk for more severe complications of COVID-19. These are undeniably difficult times, and it is important to maintain a healthy lifestyle, especially for those with predisposed health conditions and the elderly. This pandemic could last for a long time, and its long-term effect on people's lifestyle choices, such as diet, physical activity, and sleep habits, is likely to be important.

So, this review emphasizes the impact of lockdown and ongoing situation of COVID 19 on individuals eating habits, changes in daily routines like sleep pattern, screen time, stress and other lifestyle changes. Also, this review, can inspire further research in the emerging field of lifestyle medicine and its potential effect before, during, and after pandemics such as COVID-19.

\section{Dietary changes}

Most of the literature included in this review with reference to dietary habits indicates an alteration in the eating behaviours of people. Nutritional factors are interlaced with human behaviour, and emotions while playing a critical role in not just the initiation, but also the severity and duration of depression. Numerous reports support the view that stress can either increase or decrease caloric intake, and chronic stress exposure can lead to either obesity or anorexia (Rao., et al. 2008). Chopra, Ranjan, Singh., et al. (2020) found an increase in healthy meal consumption patterns and a restriction on unhealthy food items, especially among the younger population (under 30 years old). Decrease in physical activity was found, along with an increase in daily screen time, especially among men and those in the upper socioeconomic strata. In approximately onefourth of the participants, quarantine caused stress and anxiety increased by one unit. In her research on the Effect of COVID-19 on the Dietary Habits of Middle-Class Population in Mulund, Mumbai, India, Mehta [6] discovered that there was a rise in snacking habits and tea/coffee consumptions, as well as increased intakes of readyto-eat foods, decreased intakes of fruits, vegetables, legumes, and other nutritious foods, and an increase in mental tension.

Although the workload in the study population was lower than before, the mental stress increased due to reasons like financial in- 
securities and instabilities, presence of co-morbidities and stress about the ongoing pandemic. It was observed that people relied on sweet foods to satisfy their cravings, along with fried, fatty and processed foods. Also, the physical activity levels decreased in the population to an extent that could be a home for future diseases. According to the findings, by the study of Sutaria., et al. [7] in which 422 volunteers participated it was found that $23.5 \%(n=56)$ of people ate vegetables less than twice a week. Snacking between meals 1-3 times a day was recorded by $72 \%(n=304)$, with $54.9 \%$ ( $n=167$ ) exercising less or not at all than before the lockout, and $52.6 \%(n=160)$ gaining significant weight. $30.18 \%(n=64)$ of the $50.2 \%(n=212)$ who had their eating schedule modified missed at least one meal, and $22.3 \%$ had gastrointestinal problems. In their study, Basu., et al. (2020) looked at the improvements in participants' dietary habits and found that the majority of the sample population has a substantial reduction in their consumption of fast food and meat items, with $60 \%$ and $34 \%$ of participants, respectively, consuming less than before. Fresh produce and dairy product intake remained relatively unchanged for $44 \%$ and $51 \%$ of participants, respectively, and increased for $36 \%$ and $27 \%$. For $60 \%$ of participants, the amount of meals eaten per day remained unchanged, while for 24\%, it increased. After the lockdown, 13\% of participants said the amount of meals they consumed in a day decreased (The statistical data is shown by bar graph in figure 1).

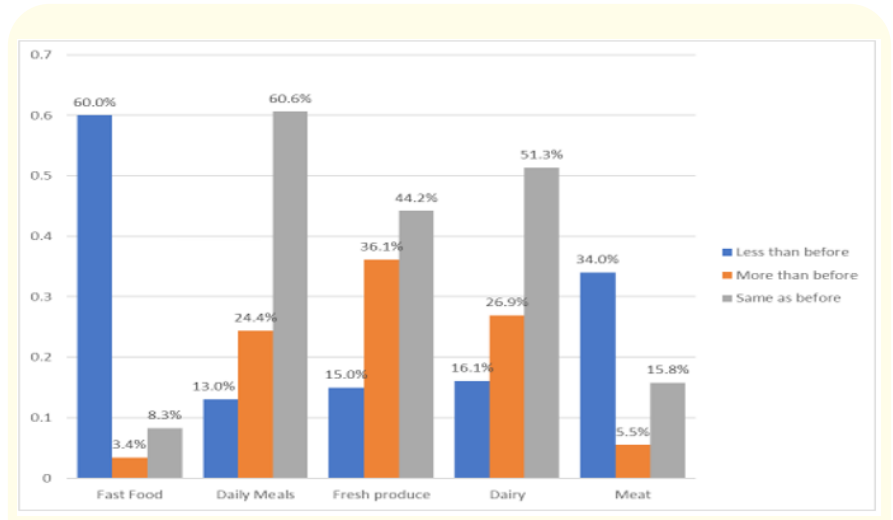

Figure 1: Bar plot depicts the change in eating habits post lockdown (Basu et al. 2020).
In the study that Badlani [8] had done on students of Pune to find out impact of COVID-19 on changing food habits founded that there has been a change in food habits due to Covid-19, more than $60 \%$ of students are now more inclined towards nutritious and healthy food as compared to unhealthy food. More than $65 \%$ students are also consuming nutritious food to improve their immunity so that they can combat the Covid-19 virus. In the study conducted on 90 subjects by Kaur., et al. [9] it was found that the percentage of respondents not consuming non-vegetarian foods significantly increased during phase one of the lockdown. There was a statistically significant correlation between non-vegetarian food intake and COVID-19 lockdown phase-1. This may be due to a shortage of fresh-cut meats, the closing of butcher shops, or personal preference. The lower consumption of non-vegetarian food by the non-vegetarians during the lockdown period may also be due to the floating speculations, myths, and facts on social media about the start of the COVID-19 outbreak from an animal market in Wuhan, China. Maximum respondents used to consume three meals and two snacks a day. On line home delivery food orders were observed to have decreased during COVID19 lockdown phase-I. During the lockdown, there was also a substantial rise in the number of respondents who said they ate salads every day.

Vitamins and minerals, play a vital role in the promotion of health and the prevention of disease. Adolescents are regarded as a nutritionally vulnerable population segment [10]. In a study done by Roy, 2020 among Adolescents and young adults it was found that mean sleeping period increased from 6.85 to 8.17 hours, average screen time increased from 3.5 to 5.12 hours, $51.9 \%$ of subjects experienced increased stress, $76.4 \%$ of subjects experienced increased food consumption, and $38.6 \%$ of subjects experienced decreased physical activity.

Immunity booster and herbs

During the unprecedented COVID-19 spread, the Ministry of AYUSH emphasised the use of preventive measures listed in Ayurveda. These include common food ingredients found in Indian kitchens 
that are beneficial to respiratory health and immunity in particular. Spices like turmeric, cumin, and coriander, as well as garlic, were readily available and easy to adapt. It is said to be helpful to use turmeric powder in hot milk every day and to drink warm water during the day. Malnutrition, insufficient sleep, physical or psychological stress, and lifestyle disorders may all weaken the immune system. Vitamin C is thought to play a role in boosting immunity and effectively preventing/treating common colds [11]. Incorporating vitamin $\mathrm{C}$-rich foods into regular diets can be an effective strategy for combating COVID-19. Vitamin C and antioxidant-rich fresh fruits and vegetables such as lemon, orange, gooseberry, grapefruit, tomatoes, papaya, broccoli, and other citrus fruits and vegetables can help us boost our immunity (Chen., et al. 2020 and Abbasi., et al. 2019). In their study, Basu., et al. (2020) found that $43 \%$ of the population surveyed did not use any form of immunity booster, accounting for $37 \%$ of all responses. Multivitamins and ginger and garlic were the most common immunity boosters, accounting for $35 \%$ of all responses (15\%). Ginger and garlic were the most frequently consumed foods among the 44 people over the age of 50 , accounting for $46 \%$ of responses. The number of people over 50 who did not take immunity boosters accounted for $23 \%$ of the answers. $42 \%$ of the 196 diseased people did not take immunity boosters, accounting for $34 \%$ of the responses. $41 \%$ of diseased participants took ginger and garlic on a regular basis, and $21 \%$ took multivitamins on a regular basis. As a result, ginger and garlic accounted for $33 \%$ of all immunity boosters used by diseased people, while multivitamins accounted for $17 \%$. Honey is a natural food with medicinal properties. Before the COVID lockdown phase-1, $7.7 \%$ of respondents said they consumed honey every day, but that number grew to $84.4 \%$ during the lockdown. (The statistical data is shown in figure 2). The number of people who take honey hasn't changed significantly. Before the COVID -19, lockdown phase-1, $87.7 \%$ of respondents did not consume honey in any form, and during the lockdown period, $12.2 \%$ of respondents did not consume honey in any form, according to the survey done by Kaur., et al. 2020. A total of 28 traditional medicine guidelines (26 government-issued Chinese and two Korean guidelines) providing treatment measures for COVID-19 were found in a study by Ang, L. 2020, who searched seven data sources for eligible traditional medicine guidelines. He claimed that there is no direct evidence of traditional formulae's efficacy in the treatment of COVID-19, and that all of them need to be clinically verified. (a) Use of various immunity boosters among all individuals
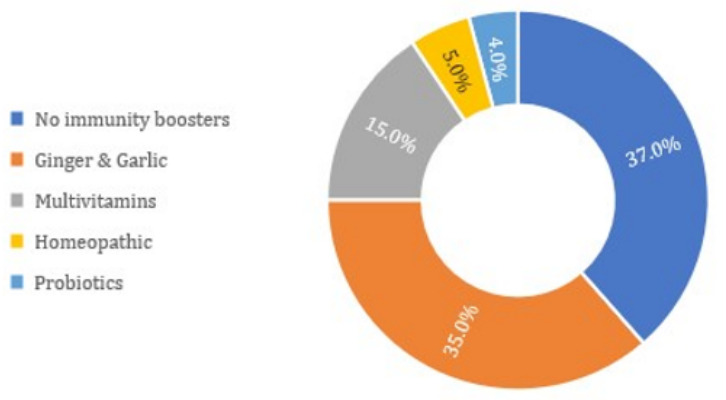

(b)Use of immunity boosters among individuals aged above 50

no immunity boosters

= Ginger \& Garlic

Multivitamins

E Homeopathic

Probiotics

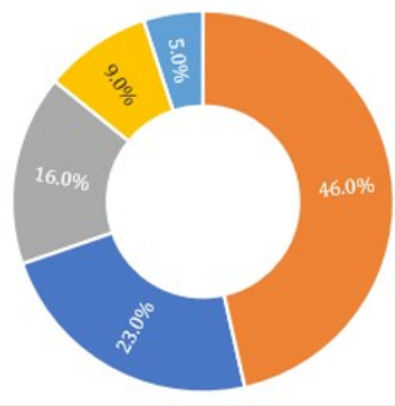

(c) Use of immunity boosters among diseased individuals

- No immunity boosters

n Ginger \& Garlic

nultivitamins

E Homeopathic

nrobiotics

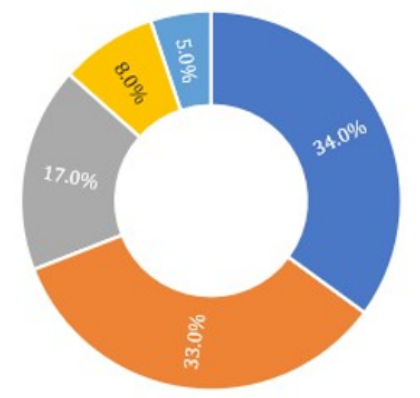

Figure 2: Donut plots shows the type of immunity boosters consumed by A) total participants B) participants above 50 years of age and C) diseased participants (Basu., et al. 2020).

\section{Physical activity}

The lockdown period saw a general increment in physical activity levels as evidenced from the studies included in our review. 
Mathur [13] reported that $54.4 \%$ adult population of India is inactive. The $65.5 \%$ inactive population is from urban areas. Women are more inactive than men. According to a WHO study from 2003, in India, $52.6 \%$ of females and $10.2 \%$ of males aged 20 and above are inactive. Inactivity raises the risk of lifestyle disorders such as stress, anxiety, and depression etc. Reading, doing household chores, listening to music, cooking, exercising, painting, and dancing were all recorded to be done by $81 \%, 7977 \%, 72 \%, 65 \%, 41 \%$, and $36 \%$ of respondents, respectively, this was stated in a study done by Basu., et al. 2020. The majority of them engaged in these activities for 1-2 hours, with exercise being the most common activity. Of all tasks done for at least 2 hours, reading had the highest frequency. (In figure 3, the statistical data is represented by a bar graph).

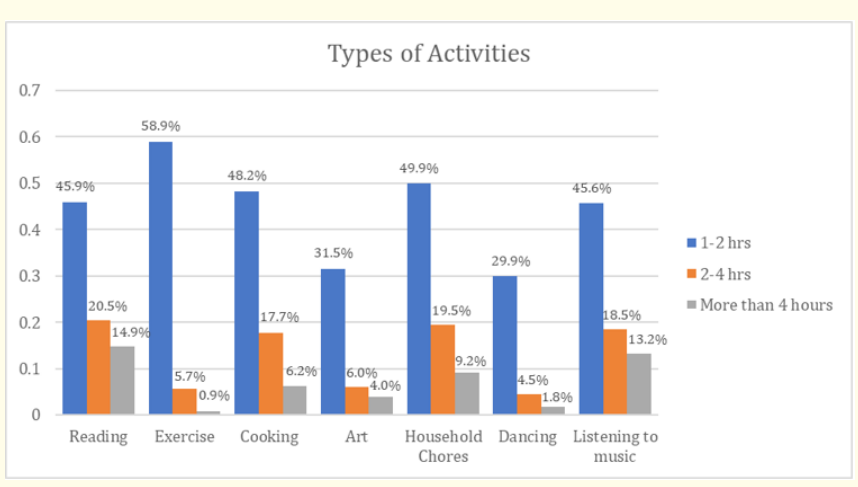

Figure 3: Bar graph represents the time devoted by participants to various activities during lockdown (Basu., et al. 2020).

Lockdown is having a profound effect beyond the virus, as demonstrated by a substantial improvement in exercise patterns, with $42 \%$ of total participants exercising at least three days per week prior to lockdown, compared to $22 \%$ after lockdown, according to the results stated by Kaur., et al. 2020 in their study. According to a study conducted by Kumar and Dwivedi (2020), it was found that only $27.7 \%$ of respondents did a physical activity on a daily basis prior to the COVID-19 lockdown situation, whereas $53.3 \%$ of respondents started doing physical activity on a daily basis after the announcement of COVID-19 lockdown phase-1. According to the key findings of the survey conducted by Chopra., et al. [12] overall physical activity has decreased in all age groups. In addition, both genders' overall physical activity declined, with men experiencing a greater significant reduction in their activity status than women.

\section{Sleeping behaviour}

Sleep deprivation may have a detrimental effect on physical and mental health, as well as the immune system's ability to combat infections (Abdurahman., et al. 2020). According to Basu., et al. 44\% of participants said their sleeping time before lockdown was mainly concentrated between $11 \mathrm{pm}$ and 1 a.m., but this proportion fell to $37 \%$ after lockdown. Those who slept between $9 \mathrm{pm}$ and $11 \mathrm{pm}$ saw a decrease of $32 \%$ to $20 \%$, while those who slept between 1a.m. and 3a.m. saw a rise of $21 \%$ to $25 \%$. The number of people sleeping after 3 a.m. increased significantly from $3 \%$ to $17 \%$.

According to Kumar and Dwivedi's [14] research, the lack of social zeitgebers, such as daily work schedules and social events, as well as changes in living conditions, have a major impact on sleep habits when they are limited. The study findings show that during lockdown, people's sleep patterns were drastically altered, with people sleeping until late in the morning. Also, results shows that only $6 \%$ of participants got up at 5 a.m. after lockdown, compared to $19 \%$ before lockdown, and $42 \%$ got up at or after 8 a.m. after lockdown, compared to just $12 \%$ before lockdown, suggesting that they spend more time in bed after lockdown. Sleep disorders have been related to a higher risk of mental illness. Anxiety, stress, and self-efficacy have also been related to sleep quality (Xiao., et al. 2020). Besides that, short sleep time has been related to suicidal thoughts in some people (Weber., et al. 2020). According to A. S. Kochhar., et al. [15], 55.3\% had trouble sleeping during the lockdown, including people who were fearful of contracting the disease and those between the ages of 35 and 50 having the most disturbance of sleep. Because of the loneliness, this may be related to high levels of anxiety and stress, suggesting poor mental health (Rajkumar, 2020).

\section{Stress}

Individuals and families grew more nervous after viewing, reading, or listening to the relentless stream of COVID-19-related news. Nutritional factors are intertwined with human actions and feel- 
ings, and they play an important role in the onset, intensity, and length of depression. Numerous studies have shown that stress can increase or decrease caloric intake, and that chronic stress can lead to obesity or anorexia (Rao., et al. 2008).

Bashu., et al. 2020 carried out a study in which participants were asked to rate their anxiety levels on a scale of 1 to 5 (1 being the least anxious and 5 being the most anxious) of which $34 \%$ reported intermediate anxiety levels. Individuals who preferred 4 and 5 on the scale made up $31 \%$ and $19 \%$ of the total sample population, respectively. Just $7 \%$ and $9 \%$ of participants selected 1 and 2 on the scale, respectively, suggesting that they had lower levels of anxiety. The data also revealed that females had higher anxiety levels than males, with $89 \%$ of females reporting intermediate or higher anxiety levels than males (77\%). Males opted for lower anxiety levels, i.e. 1 and 2 on the scale, in greater numbers than females, with $23 \%$ males and $115 \%$ females preferring these choices (The statistical data is shown in figure 4). In their analysis, Sakshi Chopra., et al. discovered that nearly one-fourth $(23.02 \%)$ of participants experienced a one-unit increase in stress. During COVID-19, however, overall social support from family and friends (20.8\%) increased. Furthermore, participants' fear of contracting the coronavirus (23.6\%), worrying about their families (20.2\%), boredom and isolation (18.2\%), and financial loss (14.7\%) were the most frequently recorded explanations for negative improvements in stress and anxiety levels during COVID-19.

\section{Lifestyle of patients with chronic diseases}

Infection is spread primarily from person to person through respiratory droplets. It is possible to take a faecal-oral route. Sputum, pharyngeal swabs, and faeces have all tested positive for the virus. SARS-CoV-2 vertical transmission has been reported [6] and confirmed by a positive COVID-19 nasopharyngeal swab. COVID-19 has a median incubation time of 5.2 days, with most patients exhibiting symptoms between 11.5 and 15.5 days. As a result, it has been requested that those who have been exposed to infection be quarantined for 14 days.

Patients with pre-existing non-communicable conditions have found it difficult to maintain a healthy lifestyle (Siddiqui., et al. 2020). Patients with coronary heart disease, diabetes, and cancer, according to Ghosh., et al. [16]) had to compromise on their physi-

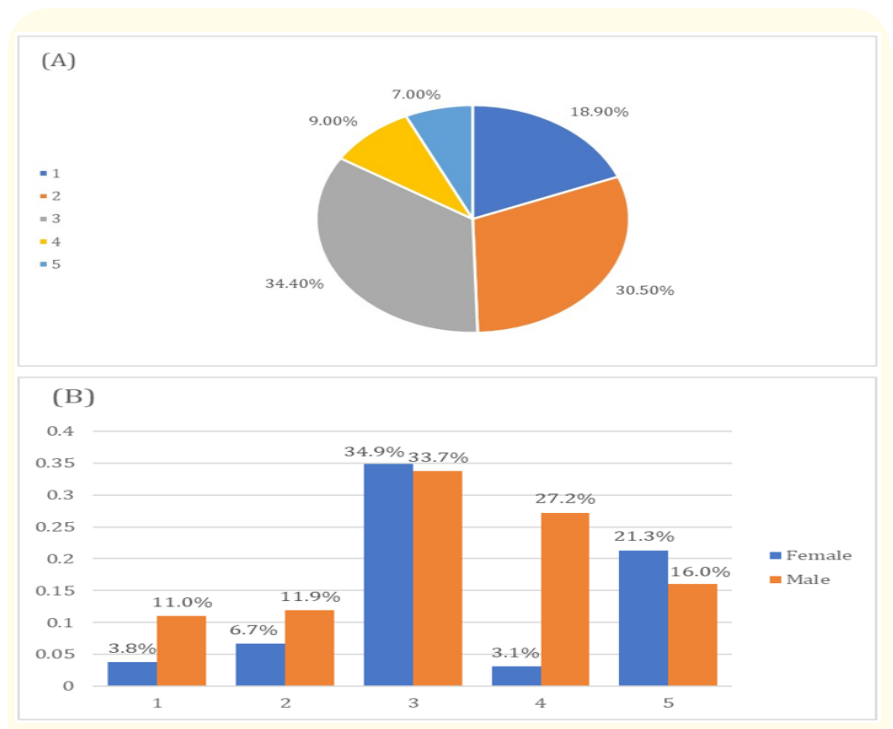

Figure 4: Figure shows A) Anxiety levels among all participants B) Anxiety levels based on gender (Basu., et al. 2020).

cal activity regimen and dietary pattern in particular. Patients who had been following up regularly before the lockdown were contacted by phone ( 45 days after the start of the lockdown) to discuss lifestyle changes, tension, and other diabetes-related issues. During 45 days of lockdown, patients with T2DM experienced a rise in refined carbohydrates, reduced exercise, decreased self-monitoring of blood glucose (SMBG), and widespread mental stress, all of which could destabilise or intensify hyperglycaemia and hypertension. Positive changes (for example, increased fruit consumption) were also observed. Nachimuthu [17] performed a pilot study, and the findings showed that $92 \%$ of subjects had Type 2 diabetes. Just $28 \%$ of the participants tested their blood glucose levels on a regular basis. During the lockdown, $80 \%$ of the participants reported that they were exercising regularly and keeping a balanced diet and the Covid-19 infection induced anxiety was found in $40 \%$ of the participants [18-25].

\section{Conclusion and Future Prospects}

The results of the study indicate a mixed effect of the preventive measures adopted to control coronavirus on the lifestyle-related behaviour with a significant improvement in regular meal consumption pattern and healthy eating behaviour and reduction in 
unhealthy food intake as positive indicators and significant reduction in physical activity and increase in sitting time, screen time and stress as negative indicators. While there were changes in eating habits, the effect was marginal. The negative effects of lifestylerelated behaviours can outweigh the benefits of a one-unit change in eating behaviour, potentially contributing to increased weight gain and metabolic complications. These results may have consequences for the development of physical activity and dietary guidelines to help people remain healthy during the COVID-19 pandemic. There were several recommendations for healthier food choices and physical activity, but it was found that during the COVID-19 lockdown phase-1 that people followed the GOI and AYUSH guidelines faithfully. The majority of Indians began to drink turmeric milk, lemon water, warm water, and integrate physical activity into their everyday routines.

The easiest and cheapest approach for health is to follow a home-cooked, healthy, and nutritious diet, exercise regularly, and integrate simple herbs into everyday routines. The findings showed that some changes had occurred, owing primarily to the lack of essential foods such as fruits and vegetables. Other factors that led to the observed changes included changes in financial situations, cravings, and so on. Increased snacking habits and tea/coffee consumption, increased intakes of ready-to-eat foods, decreased intake of fruits, vegetables, legumes, and other healthy foods, and an increase in mental stress were the most commonly observed changes. Despite the fact that the workload was lower than before, mental stress increased as a result of factors such as financial insecurity and instabilities, the presence of comorbidities, and the ongoing pandemic. People were found to rely on sweet foods, as well as fried, fatty, and refined foods, to fulfil their cravings. Furthermore, the population's level of physical activity declined to the point that it could serve as a breeding ground for potential diseases. Clinical trials focused on the connections between diet and COVID 19 are, however, missing. More research is required to fully comprehend the change in diet trends after the COVID-19 pandemic.

\section{Bibliography}

1. WHO. "Coronavirus disease (COVID-19) outbreak" (2020).

2. Bharati Y. "Aarogya Setu App: Here's everything you need to know | GQ India". Retrieved June 13 (2020).

3. Ammar A., et al. "Effects of COVID-19 Home Confinement on Eating Behaviour and Physical Activity: Results of the ECLBCOVID19 International Online Survey". Nutrients 12.6 (2020): 1583 15ita 3 tion: Jyoti Singh and Nikita Wadhawan. "Im
Scientific Nutritional Health 5.5 (2021): 96-103.
4. Kunstler B., et al. "Physical activity and sedentary behaviourduring the COVID-19 pandemic: An Australian population study". (2020).

5. Muscogiuri G., et al. "Nutritional recommendations for CoVID-19 quarantine”. European Journal of Clinical Nutrition 14 (2020): 1-2.

6. Mehta V. "The Impact of COVID-19 on the Dietary Habits of Middle-Class Population in Mulund, Mumbai, India". Section: Coronavirus Article Id: 82, Version: 1. (2020).

7. Sutaria M., et al. "COVID 19 and its effect on nutrition". International Journal of Community Medicine and Public Health 7.10 (2020): 4112-4117.

8. Badlani H. "Impact of COVID-19 on changing food habits by the students in Pune". Journal of University of Shanghai for Science and Technology 22 (2020): 607-623.

9. Kaur B., et al. "Impact of COVID-19 lockdown on the Dietary Pattern and Physical Activity of People". Journal of Humanities and Social Sciences Research 2 (2020): 205-216.

10. Tak J and Wadhawan N. "Assessment of dietary micronutrient deficiency among adolescent girls". Food Science Research Journal 7 (2016): 340-344.

11. Douglas R M., et al. "Vitamin C for preventing and treating the common cold". Cochrane Database System Review (2004).

12. Chopra., et al. "Impact of COVID-19 on lifestyle-related behaviours- a cross-sectional audit of responses from nine hundred and ninety-five participants from India". Diabetes and Metabolic Syndrome: Clinical Research and Reviews (2020): 20212030.

13. Mathur P and Mascarenhas L. "Lifestyle diseases, Keeping fit for a better tomorrow". Indian Journal of Medical Research 149.1 (2019): 129135. 2019.

14. Kumar M and Dwivedi S. "Impact of Coronavirus Imposed Lockdown on Indian Population and their Habits". International Journal of Science and Healthcare Research 5.2 (2020): 88-97.

15. Kochhar A., et al. "Impact of lockdown on mental health during Covid-19 pandemic”. Asian Journal of Psychiatry 293 (2020).

16. Ghosh., et al. "Effects of nationwide lockdown during COVID-19 epidemic on lifestyle and other medical issues of patients with type 2 diabetes in north India". Diabetes and Metabolic Syndrome: Clinical Research and Reviews (2020): 917-920. 
17. Nachimuthu S., et al. "Coping with diabetes during the COVID - 19 lockdown in India: Results of an online pilot survey". Diabetes and Metabolic Syndrome: Clinical Research and Reviews 14.4 (2020): 579-582.

18. “Covid-19: Turmeric milk to yoga, Ayush Ministry issues selfcare guidelines". The Tribune (2020).

19. Di Renzo L., et al. "Eating habits and lifestyle changes during COVID-19 lockdown: an Italian survey". Journal of Translational Medicine 18 (2020): 229.

20. Pandit M., et al. "Impact of lockdown due to COVID-19 outbreak: lifestyle changes and public health concerns in India". The International Journal of Indian Psychology 8.2 (2020): 1386-1411.

21. Narayanan L., et al. "Impact of lockdown due to COVID-19. outbreak: lifestyle changes and public health concerns in India" (2020).

22. Tripathy P and Wadhawan N. "Assessment of food and nutrient intake of women beneficiaries ofmgnrega scheme in Rajasthan". Agricultural Research Journal 55.1 (2018): 134-139.

23. Pietrobelli A., et al. "Effects of COVID-19 Lockdown on Lifestyle Behaviors in Children with Obesity Living in Verona, Italy: A Longitudinal Study". Obesity 28 (2020): 1382-1385.

24. Ruiz-Roso M B., et al. "Covid-19 Confinement and Changes of Adolescent's Dietary Trends in Italy, Spain, Chile, Colombia and Brazil". Nutrients 12.6 (2020): 1-18.

25. Roy S., et al. "Impact of COVID-19 Pandemic led lockdown on the lifestyle of Adolscents and young adults" (2020).

\section{Assets from publication with us}

- Prompt Acknowledgement after receiving the article

- Thorough Double blinded peer review

- Rapid Publication

- Issue of Publication Certificate

- High visibility of your Published work

Website: www.actascientific.com/

Submit Article: www.actascientific.com/submission.php

Email us: editor@actascientific.com

Contact us: +919182824667 